\title{
UNA LECCIÓN SOBRE EL LIBERALISMO ANARQUISTA DE R. NOZICK ${ }^{1}$
}

\author{
José MARTínez De PisÓn CAVERo \\ CATEDRÁtico de Filosofía del DERECho \\ UNIVERSIDAD DE LA RIOJA \\ jose.mezdepison@unirioja.es
}

SUMARIO: I. UNA TEORÍA FUERTE DEL LIBERALISMO NEOLIBERAL O LIBERTARIO. II. CONCEPCIÓN DEL HOMBRE COMO FIN EN SÍ MISMO. III. LA TEORÍA DE LA JUSTICIA: UNA RETÓRICA DEL DERECHO DE PROPIEDAD. IV. RECONSTRUCCIÓN HISTÓRICO-ANTROPOLÓGICA DE LA HISTORIA INSTITUCIONAL Y POLÍTICA. V. JUSTICIA RETRIBUTIVA VS. JUSTICIA DISTRIBUTIVA. VI. ¿EL ESTADO MÍNIMO COMO LA UTOPÍA DEL ANARQUISMO LIBERTARIO? VII. ANÁLISIS CRÍTICO. VIII. BIBLIOGRAFÍA.

RESUMEN: R. Nozick fue, dentro del liberalismo, el primer gran contradictor de J. Rawls. Frente a la apuesta de una "justicia como equidad" claramente distributiva e igualitarista, Nozick enarboló un liberalismo anarquista centrado en el poder del mercado y de sus instrumentos reguladores con un Estado reducido a la más mínima expresión. Por ello, la teoría de R. Nozick constituye una interesante y provocadora versión del liberalismo que ha atraído a numerosos políticos y pensadores.

PALABRAS CLAVE: Robert Nozick, liberalismo, anarquismo liberal, libertarismo, teoría de la justicia.

\section{A LESSON ON ANARCHIST LIBERALISM FROM R. NOZICK}

ABSTRACT: R. Nozick was, within liberalism, the first great contradictor of J. Rawls. Faced with the bet of a clearly distributive and egalitarian "justice as fairness", Nozick promoted an anarchist liberalism centered on the power of the market and its regulatory instruments with a State reduced to the most minimal expression. For this reason, R. Nozick's theory constitutes an interesting and provocative version of liberalism that has attracted numerous politicians and thinkers.

KEY WORDS: Robert Nozick, liberalism, liberal anarchism, libertarism, theory of justice.

\footnotetext{
${ }^{1}$ Con este trabajo sigo la labor de recuperación de las lecciones que preparé e impartí en la asignatura de Filosofía Política en la Universidad de La Rioja durante los cursos 2000-2001 a 2005-2006. Como en el caso de la lección sobre J. Rawls se trata, en realidad, de una reelaboración de los materiales con los que estuvimos trabajando en el aula. Espero que, además de recordatorio, estas páginas sirvan de introducción a la obra y pensamiento de R. Nozick.
} 


\section{Una teoría fuerte del liberalismo neoliberal o libertario}

R. Nozick (Nueva York, 1938), profesor de Filosofía en la Universidad de Harvard, fue uno de los primeros en responder y criticar al igualitarismo liberal de J. Rawls y su $A$ Theory of Justice, al publicar en 1974 Anarquia, Estado y Utopia (Anarchy, State and Utopia ${ }^{2}$ ). Es considerado como una de las mentes "más versátil, punzante y ágil”, con amplios conocimientos filosóficos desde que, según ha reconocido él mismo, todavía adolescente se interesara por la República de Platón. Después de Anarcby, State and Utopia, su primer libro, ha publicado Pbilosophical Explanations (1981), sobre la naturaleza del conocimiento, el yo, la voluntad libre y la ética; The Examined Life (1990), un libro en el que reflexiona sobre el amor, la felicidad, la creatividad, el bien, el mal y el holocausto; La Naturaleza de la racionalidad (1993), en el que estudia los modelos de acción racional y elabora una teoría de la creencia. Después publicó Socratic Puž̨les (1997) y, antes de fallecer el 22 de enero de 2002, aún dio tiempo de que saliese de las prensas The Structure of the Objetive World (2001).

$\mathrm{Al}$ explicar esta trayectoria vital, puede entreverse una primera diferencia entre R. Nozick y J. Rawls. Este último, antes y después de $A$ Theory of Justice, se dedicó siempre a elaborar o reelaborar su teoría de la justicia como equidad ${ }^{3}$. Si recordamos lo ya visto, durante un tiempo, desde finales de los años 50 del siglo pasado, Rawls se dedicó a presentar y reflexionar sobre sus nuevas e importantes propuestas hasta confeccionar su magna obra. Después, tras las fuertes críticas, pasó un tiempo respondiendo a sus críticos hasta que fue más allá presentando una nueva versión de la justicia como equidad. En última instancia, sus publicaciones y su larga trayectoria intelectual giraron en torno a los temas y problemas planteados en su filosofía moral, política y social desde el inicio de su carrera académica.

Por el contrario, R. Nozick empezó su proyección intelectual con el texto que va a ser objeto de estudio, Anarquía, Estado y Utopía, y posteriormente, como puede entreverse por los títulos de las publicaciones antes citadas, sus temas y problemas, sus explicaciones y argumentaciones derivarían más hacia cuestiones filosóficas en las que se encontraba más cómodo ${ }^{4}$. No puede perderse de vista, incluso, que, a pesar del éxito que tuvo, Nozick no estaba muy satisfecho con su primera magna obra y que, aunque no llegara a repudiarla, sí que puso de manifiesto sus cambios de opinión en las cuestiones tratadas 5 . En todo caso, no cabe duda de que Anarquía, Estado y Utopía tuvo un importante

\footnotetext{
2 R. NOZICK, Anarquía, Estado y Utopía, trad. de R. Tamayo, México, Fondo de Cultura Económica, 1988. En estas páginas, se utilizará el acrónimo $A S U$ para referirse a esta obra y, a continuación, se añadirá el número de página de referencia.

3 J. RAWLS, Teoría de la Justica, trad. de M. D. González, Madrid, FCE, 1979.

${ }^{4}$ R. Nozick fue un filósofo de amplio espectro que, como puede verse por su obra no política, se preocupó por cuestiones que rara vez son objeto de reflexión o, como gustaba el autor, de "explicación". De hecho, como afirma en las primeras páginas de Explicaciones filosóficas, Nozick no era defensor de la moda de la "argumentación", pues entendía que, en todo proceso de argumentación, hay un punto de coacción y de imposición: el que demuestra más habilidad, gana y el perdedor está obligado a aceptar las tesis contrarias y asumirlas. Por eso, prefería referirse a la filosofía como proceso de "explicación” y no de imposición. Vid. la "Introducción” de Explicaciones filosóficas, trad. J. Marqués, Innisfree, pp. 5-19.

${ }^{5}$ Nozick escribió en la introducción de Meditaciones sobre la vida lo siguiente: "Habiendo escrito un libro de filosofía política que delineaba una perspectiva particular que hoy no me satisface -más adelante diré algunas palabras sobre esto-, soy muy consciente de la dificultad de rehuir un pasado intelectual. En muchas conversaciones otros desean que yo conserve la posición 'libertaria' de ese joven, aunque ellos mismos la rechazan y quizá preferirían que nadie la hubiera sostenido jamás”. R. NOZICK, Meditaciones sobre la vida, $3^{\mathrm{a}}$ ed. trad. de C. Gardin, Barcelona, Gedisa, 2018, p.15.
} 
impacto y ha sido, desde su publicación, una obra de referencia en sectores relevantes de la política y economía estadounidense y en la europea ${ }^{6}$.

R. Nozick es un liberal libertario 7 . O sea, un liberal que, tras la pantalla de la reivindicación del liberalismo clásico, esconde un pensamiento conservador que, además, reacciona pasionalmente ante la versión del liberalismo apadrinada por Rawls. Eso quiere decir que su pensamiento -al menos, inicialmente- es una reacción contra el igualitarismo, la justicia distributiva y, sobre todo, contra todo lo que supone el Estado social o del bienestar: su intervencionismo, las políticas asistenciales y sociales, el sistema fiscal progresivo, etc. El Estado como violador de los derechos más esenciales del individuo es el objeto de discusión de Anarquía, Estado y Utopía. No es necesario un Estado activo para que se materialicen los derechos individuales; más bien, por el contrario, es necesario un "Estado menos ambicioso". Un Estado mínimo con funciones limitadas. En realidad, Nozick pretende una reivindicación del viejo Estado liberal. Pero, incluso, en este caso, las funciones que le asigna son menores que en dicho modelo. Así lo describe en el Prefacio de su libro: "Mis conclusiones principales sobre el Estado son que un Estado mínimo limitado a las estrechas funciones de protección contra la violencia, el robo y el fraude, de cumplimiento de contratos, etcétera, se justifica; que cualquier Estado más extenso violaría el derecho de las personas de no ser obligadas a hacer ciertas cosas y, por tanto, no se justifica; que el Estado mínimo es inspirador, así como correcto. Dos implicaciones notables son que el Estado no puede usar su aparato coactivo con el propósito de hacer que algunos ciudadanos ayuden a otros o para prohibirle a la gente actividades para su propio bien o protección"8.

El Estado mínimo y la obra de R. Nozick implican así todo un desafío al éxito tenido por la obra de J. Rawls y también al intento de un cierto sector del liberalismo por limitar los efectos más perjudiciales del liberalismo y del capitalismo incorporando alguna de las tesis de la socialdemocracia. Al mismo tiempo, esta propuesta es un intento de mostrar que se puede justificar un modelo anarquista basado en el liberalismo sin que se violen los derechos individuales. Una sociedad liberal anarquista en la que el derecho de propiedad y la economía de mercado sean el eje en torno al cual gira la vida social; una sociedad anarquista liberal en la que no sea necesario el Estado.

\footnotetext{
${ }^{6}$ Dieterlen escribe dos reflexiones relevantes sobre Anarquía, Estado y Utopía: por un lado, que, junto con Teoría de la Justicia de J. Rawls, “son las dos obras más importantes en el pensamiento anglosajón”; y que, por otro, “el libro de Nozick no es un libro fácil; en él encontramos, en palabras del propio autor, argumentos elaborados, tesis sorprendentes, enigmas, condiciones estructurales abstractas, desafíos para encontrar otras teorías que convengan a una clase especial de casos, conclusiones alarmantes, etc. Estos elementos dificultan la presentación de las tesis, pero, al mismo tiempo, constituyen un reto para el lector". P. DIETERLEN, "La filosofía política de Robert Nozick", Revista Mexicana de Ciencias Políticas y Sociales, vol. 37, no 150, 1998, p. 123.

${ }^{7}$ Sobre el libertarismo, puede verse W. KYMLICKA, Filosofía politica contemporánea. Una introducción, trad. de R. Gargarella, Barcelona, Ariel, 1995, pp. 109-178, y F. R. TESÓN “Libertarismo” en I. GONZÁLEZ RICOY y J. QUERALT (eds.), Razones públicas. Una introducción a la Filosofía Política, Barcelona, Ariel, 2021, pp,117-139. Como se afirme en este último trabajo, el libertarismo "descansa en tres pilares que se refuerzan mutuamente. Los libertarios proponen: 1) amplias libertades individuales, tanto civiles y políticas como económicas; 2) mercados libres; y 3) un rol modesto para el Estado”. A su vez, distingue, según las funciones atribuidas al Estado, entre libertarios anarquistas, libertarios lockeanos y libertarios kantianos. Incluye a R. Nozick en la categoría de libertarios lockeanos dado que atribuye al Estado la defensa frente al exterior y la protección de los derechos individuales. También sobre el libertarismo y el anarquismo liberal, vid. F. SCHWEMBER y D. LOEWE, "Si el mundo fuera completamente justo: Nozick y los límites de la utopía liberal”, Revista de Estudios Políticos, 182, 2018, pp. 43-49.

8 ASU, p. 7.
} 
La teoría de Nozick es también una teoría fuertemente individualista9. Son precisamente los derechos individuales -sus derechos naturales al estilo de J. Locke- los que se alzan como límites al activismo del Estado. En Anarquia, Estado y Utopía existe una constante tensión individuo-Estado en la que el primero sale siempre triunfante. Nozick es muy dado a exagerar en sus frases, a acompañar sus opiniones con una retórica fuerte, pero al mismo tiempo muy gráfica, pues refleja el trasfondo de sus opiniones. Así, es obligado transcribir la frase con la que inicia el libro en la que se refleja la tensión mencionada y, sobre todo, su individualismo: "Los individuos tienen derechos, y hay cosas que ninguna persona o grupo puede hacerles sin violar los derechos. Estos derechos son tan firmes y de tan largo alcance que surge la cuestión de qué pueden hacer el Estado y sus funcionarios, si es que pueden algo. ¿Qué espacio dejan al Estado los derechos individuales?" ${ }^{10}$. El libro gira en torno a esta pregunta y su respuesta que, como puede suponerse, es nada. Ambas resumen el pensamiento libertario de R. Nozick.

Al mismo tiempo, R. Nozick acepta el reto de Rawls de recuperar y reformular las bases ilustradas del liberalismo. Así, en defensa de su anarquismo liberal y del Estado mínimo esboza una reconstrucción del primigenio estado de naturaleza con el que los contractualistas querían explicar el origen de la sociedad y justificar el Estado. Nozick, en este punto, sigue a J. Locke. De ahí que su diseño del estado de naturaleza tiene particularidades que le diferencian radicalmente de la propuesta de Rawls. Primero, hay que indicar que Nozick no es un contractualista: el paso del estado de naturaleza al estado social no se produce a través de un contrato, sino que es el resultado de un proceso histórico, de la misma evolución que ha sido la nota común del desarrollo de la humanidad. Además, el estado inicial no es una situación o hipotética al estilo de Rawls; no es un constructo imaginado y diseñado para lograr un acuerdo sobre principios de justicia. Nozick pretende convencernos de que "su" estado de naturaleza fue un momento determinado "real", históricamente dado y así lograr un fundamento fuerte para su teoría del Estado mínimo. Por último, ese estado de naturaleza no es un constructo pensado como un procedimiento cuyo objetivo es entablar un diálogo y alcanzar un acuerdo básico para la convivencia. Por el contrario, pretende convencernos de que en él se desarrollan las fuerzas de la naturaleza y las capacidades naturales de los individuos sin cortapisas. Que actuamos tal como somos y que eso es lo que hace que no quepa más forma estatal que el Estado mínimo. Como afirma Kymlicka, éste es el reto y el punto fuerte de su teoría: si Nozick logra convencernos de que realmente la evolución de la humanidad se ha producido según sus explicaciones, su teoría adquiere un refuerzo argumentativo y moral por encima de otras teorías, incluida la de Rawls.

Nozick afirma que son J. Locke, I. Kant y A. Smith la fuente de inspiración de su anarquismo liberal. Sobre todo, su teoría está muy influida por J. Locke del que toma dos elementos muy importantes. Por un lado, su descripción del estado de naturaleza es una variación del descrito por Locke en su Two Treatise of Government. Toma como punto de partida las explicaciones de Locke para superar sus inconvenientes y, sobre todo, para mostrar que, a partir de esa descripción, es posible la evolución hacia formas societarias sin un contrato social ${ }^{11}$. Además, utiliza de las aportaciones de Locke su teoría de los derechos naturales: los derechos atribuidos a los individuos -derechos intocables e inalienables- son los derechos naturales de Locke y, sobre todo, el derecho de propiedad.

\footnotetext{
${ }^{9}$ Como afirma Grondona, en el pensamiento de Nozick, "la dignidad del hombre es tal, que no puede aceptar que de ninguna manera se violen sus derechos". M. GRONDONA, Los pensadores de la libertad. De John Locke a Robert Nozick, Buenos Aires, Editorial Sudamericana, 1986, p. 162.

10 ASU, p. 7.

11 Vid. J. LOCKE, Ensayo sobre el gobierno civil, trad. de A. Lázaro, Madrid, Aguilar, 1981, pp. 5-14.
} 
Asimismo, Nozick es un autor kantiano en la medida que acepta y revisa el concepto de persona moral como fin en sí mismo de Kant. Se puede decir que la teoría de Nozick postula un individualismo fuerte resultado de la conjunción del concepto kantiano de persona y la asignación de los derechos lockeanos. Por último, es visible la influencia de A. Smith en sus explicaciones. Para Nozick, la evolución desde el estado de naturaleza hasta el Estado mínimo es resultado de la acción de la "mano invisible". "Una explicación del tipo de la mano invisible" justifica la evolución desde el estado de naturaleza y el paso por diferentes fases -las asociaciones de protección y el Estado ultramínimo- hasta el Estado mínimo ${ }^{12}$.

En resumen, la teoría de Nozick gira en torno al Estado mínimo y a la justificación de que, respecto a las funciones estatales, no puede irse más allá. Para lograr este objetivo, Anarquía, Estado y Utopía se estructura en tres partes. Cada una de ellas con una finalidad. La primera está dedicada precisamente a justificar al Estado mínimo y a sus limitadas funciones de protección contra el robo, el fraude, el cumplimiento de contratos, etc. Su objetivo es convencer al espectro anarquista de la validez del Estado mínimo. Como afirma Gargarella, "frente al anarquista, Nozick va a tratar de demostrar que es posible llegar al Estado mínimo sin incurrir en violaciones de derechos, y además que dicha trayectoria -desde el estado de naturaleza al Estado mínimo no sólo es posible y legítima, sino además moralmente necesaria"13.

La segunda "sostiene que ningún Estado más extenso puede justificarse"; por ello, intenta deslegitimar cualquier forma de Estado que se exceda de estas funciones, principalmente los que tienen por objetivo materializar un ideal de justicia distributiva ${ }^{14}$. En esta parte desarrolla su concepción de la justicia como una entitlement theory, esto es, como una teoría sobre los derechos adquiridos con justo título. Esta parte tiene por objetivo mostrar que cualquier exceso en el poder del Estado es injusto. La conclusión es que la justicia como equidad de Rawls es un ejemplo de injusticia. En la última parte, trata la cuestión de la utopía; pretende mostrar que el Estado mínimo es la utopía misma. Como afirma él mismo, en la tercera parte sostiene "que, además de ser el único correcto, el Estado mínimo no deja de ser sugestivo".

\section{Concepción del hombre como fin en sí mismo.}

Los liberales libertarios en general y Nozick en particular tienen en muy alta estima el papel de los derechos en la caracterización del concepto de individuo que emplean en su teoría. En el caso de Nozick hay una apuesta clara por perfilar un concepto fuerte de persona que justifique su anarquismo liberal. Con ello trata de hallar un sólido fundamento moral para su teoría. Puede decirse por ello que la validez, el éxito o fracaso del liberalismo libertario de Nozick depende en buena medida del éxito o fracaso en la realización esta tarea.

En síntesis, como bien ha visto Kymlicka, el anarquismo liberal de Nozick se sustenta en la idea de que los individuos tienen derechos donde tener "derechos" significa que "uno es dueño de sí mismo", esto es, que los individuos tienen "un derecho incuestionable para disponer libremente" de sí mismo, de sus cualidades y de sus bienes del modo en que uno lo considere convenientemente, en tanto ello no implique el uso de la fuerza. Su fundamento moral es atribuido al hecho de que, como

\footnotetext{
12 ASU, pp. 30 y ss., p. 62 y pp. 121-122.

${ }^{13}$ R. GARGARELLA, Las teorías de la justicia después de Rawls, Un breve manual de filosofía, Barcelona, Paidós, 1999, p. 46.

14 ASU, p. 9.
} 
individuos, somos dueños de nosotros mismos; somos moralmente personas en tanto que somos propietarios de nosotros mismos ${ }^{15}$.

Como pone de manifiesto Gargarella, esta tesis de los derechos tiene unos perfiles muy claros que enlazan con las primeras formulaciones de inspiración liberal, en particular, como podemos imaginar, con la de Locke: "Los derechos asumidos por Nozick se distinguen por tres características fundamentales: son sólo derechos negativos, actúan como restricciones laterales frente a las acciones de los demás y son exhaustivos" 16 . No le falta razón a Gargarella cuando afirma que, de esta consideración, se infieren el resto de explicaciones sobre el derecho de propiedad y la evolución del estado de naturaleza al Estado mínimo ${ }^{17}$.

Así pues, esta concepción fuerte de los derechos individuales tiene importantes implicaciones. En primer lugar, según Nozick, tenemos un derecho ilimitado a nuestras cualidades personales y a nuestros bienes ${ }^{18}$. Por ello, los individuos pueden gozar de sí mismos y de sus posesiones sin ninguna cortapisa externa. Por supuesto, sin injerencia, ni obstaculización de los poderes públicos. Los liberales igualitarios, como Rawls y Dworkin, también mantienen que los individuos pueden disfrutar de estos derechos; sin embargo, mantienen además que es injusto que la gente se muera de hambre, no puedan tener acceso a una buena salud o a una vida digna. Por ello, estos últimos consideran y defienden el efecto benefactor de los sistemas impositivos y de las actuaciones del Estado en sanidad, educación, cultura, etc.

Por el contrario, para Nozick y los libertarios, los impuestos son una forma legal de usurpación y una injerencia injustificada en los derechos y en la libertad individual. Claramente, Nozick se pronuncia en contra de la "justicia distributiva" enarbolando la bandera de su teoría retributiva para la cual no se requiere un Estado con poderes: "El Estado mínimo es el Estado más extenso que se puede justificar. Cualquier Estado más extenso viola los derechos de las personas" 19 .

Además, la idea de que el carácter moral de la persona se basa en el principio de "ser dueño de uno mismo" es la piedra angular del anarquismo libertario de Nozick, de su defensa de un derecho absoluto de la propiedad y del Estado mínimo. El origen de esta idea se encuentra en Kant en su concepción de la persona como "fin en sí mismo" -que, por cierto, también es utilizada por Rawls-. La concepción de Nozick se encuentra resumida en la frase ya transcrita de que "los individuos tienen derechos, y hay cosas que ninguna persona o grupo puede hacerles sin violar sus derechos" ${ }^{20}$. Por ello, la sociedad debe respetar esos derechos porque "ellos reflejan el principio kantiano subyacente, de que los individuos son fines, no simplemente medios; no pueden ser sacrificados o usados, sin su

\footnotetext{
${ }^{15}$ KYMLICKA, Filosofía política contemporánea, cit., p. 118 y ss.

16 GARGARELLA, Las teorías de la justicia después de Rawls, cit., p. 47.

17 Paradójicamente, Dieterlen escribe: "Uno de los aspectos que más sorpresa causa cuando leemos Anarquía, Estado y Utopía es que no hay ninguna teoría acerca de los derechos, ni de cómo surgen, ni de cuál jerarquía tienen, ni de su fundamentación. Lo único que hace Nozick es describir cómo funcionan como partes componentes de una teoría moral. Los derechos funcionan como restricciones morales indirectas, es decir, los derechos determinan lo que no debemos hacer" y son expresión de la segunda formulación del imperativo categórico kantiano, del individuo como fin en sí mismo. P. DIETERLEN; "La filosofía política de Robert Nozick", Revista Mexicana de Ciencias Políticas y Sociales, cit., p. 125.

18 NOZICK dedica unas páginas a criticar la tesis rawlsiana de que las habilidades y los dones naturales personales deben ponerse al servicio de los demás. Para NOZICK, las capacidades naturales deben ser utilizadas de forma irrestricta por parte de su titular. Sobre este debate, puede verse USA, pp. 210 y ss.

19 ASU, p. 153.

${ }^{20}$ ASU, p. 7
} 
consentimiento, para alcanzar otros fines. Los individuos son inviolables" 21 . Y una teoría fuerte sobre el carácter moral de los individuos -como dueños de sí mismos- exige una teoría fuerte sobre los derechos tanto porque así se reafirma su existencia individual como porque establecen límites a lo que otros pueden hacer y a lo que se nos puede exigir como individuos. Respetar, pues, los derechos de las personas no son sino resultado de la concepción de las personas como fin en sí mismo, y no como un instrumento o medio. "Que se nos trate con respeto, mediante el respeto de nuestros derechos, nos permite, individualmente o con quien nosotros escojamos, decidir nuestra vida, y alcanzar nuestros fines y nuestra concepción de nosotros mismos, tanto como podamos, ayudados por la cooperación voluntaria de otros que posean la misma dignidad. ¿Cómo osaría cualquier Estado o grupo de individuos hacer más, o menos?"’22.

Sin duda, la teoría de Nozick, su fuerte individualismo y su teoría de los derechos como expresión del principio de "ser dueño de uno mismo" son muy atractivos, sobre todo para quienes viven en sociedades desarrolladas y de tradición individualista, pues supone una reafirmación de uno de los presupuestos de la cultura occidental. Puede decirse incluso que estos postulados no se separan de lo defendido por otras versiones del liberalismo. Pero, la cuestión ulterior es preguntarnos por los derechos atribuidos a las personas morales. Aquí empiezan las diferencias. Para Nozick, el derecho principal, incluso el que confiere el carácter moral de personas, es el derecho de propiedad. Somos dueños de nosotros mismos en la medida en que somos propietarios, en la medida que tenemos un derecho de propiedad sobre nosotros mismos y sobre nuestras cosas. En esto, existe una clara diferencia en relación con la teoría de Rawls, para quien es cierto que tenemos un derecho sobre nosotros y nuestras cosas, pero también lo tenemos sobre una porción de los bienes y recursos sociales. Lo suficiente como para gozar de una vida digna.

Nozick considera que Rawls yerra al defender una justicia redistributiva. La redistribución rawlsiana, en su opinión, no expresa fielmente el ideal kantiano de persona como fin en sí mismo porque al final toma a algunas personas y a sus recursos como medios para lograr una vida digna que mejore la situación de otros. "Dado que yo tengo derechos a ser dueño de mí mismo, los naturalmente menos favorecidos no tienen ninguna pretensión legítima sobre mí o mis circunstancias favorables. Lo mismo es cierto respecto de todas las otras intervenciones coercitivas en los intercambios de libre mercado. Sólo el capitalismo sin restricciones puede reconocer plenamente la propiedad sobre mí mismo", tal y como resume Kymlicka la posición de Nozick ${ }^{23}$.

Precisamente, el que consideremos a las personas como dueñas de sí mismas es, según Nozick, fundamental para que sean tratadas como seres iguales. El principio de "ser dueños de sí mismos" es, por tanto, la pieza clave para comprender el concepto de igualdad de Nozick: somos iguales en la consideración de ser dueños de nosotros mismos. Por supuesto, esta consideración se mueve en el plano abstracto; para nada tiene en cuenta la situación real de cada uno, las desigualdades económicas y sociales, y su importancia para que los individuos puedan tener expectativas, confeccionar sus planes de vida y satisfacer sus intereses.

\footnotetext{
21 ASU, p. 43.

22 ASU, p. 319.

${ }^{23}$ KYMLICKA, Filosofía política contemporánea, cit., p. 122.
} 


\section{La teoría de la justicia: una retórica del derecho de propiedad}

Las reflexiones de Nozick sobre el derecho de propiedad cobran así una especial importancia. Por un lado, son la piedra angular de su concepción de la persona como dueña de sí misma; por otro, reflejan su ideal de igualdad. En realidad, su concepción sobre el derecho de propiedad concreta la teoría sobre la justicia de Nozick que éste enarbola contra la justicia como equidad de Rawls.

Sin embargo, es una teoría que plantea de partida serias dudas. La principal, como afirma Kymlicka, es la de mostrar que efectivamente el ser dueños de sí mismo conlleva indefectiblemente la reafirmación de un derecho absoluto e intocable de propiedad. La segunda, que ello sea un modelo de igualdad. Finalmente, que el modelo de capitalismo sin restricciones y el Estado mínimo sean el mejor contexto económico, social e institucional en el que las personas puedan ser dueñas de sí mismas $^{24}$.

La teoría sobre la justicia y sobre la propiedad de Nozick -la justicia retributiva frete a la justicia distributiva- recibe el nombre de teoría del titulo justo (entitlement theory) ${ }^{25}$. Para Nozick, que los individuos sean dueños de sí mismo quiere decir que tienen poderes y que pueden ejercerlos. Sólo que en el mercado la ejecución de tales poderes implica derechos legales. Los intercambios en el mercado no son posibles sin una clarificación del derecho de propiedad y de los modos de adquisición de los títulos que legitiman la propiedad sobre los bienes. Por ello, resultan cruciales sus explicaciones sobre estas cuestiones.

Su teoría de la justicia, esto es, su teoría del título justo y, por tanto, la base del derecho de propiedad se resume en una máxima: "Una distribución es justa si surge de otra distribución justa a través de medios legítimos". O, dicho de otra manera: "Cualquier cosa que surja de una situación justa por pasos justos es justa en sí misma". Pues, como afirma Nozick: "Si el mundo fuera completamente justo, las siguientes definiciones inductivas cubrirían exhaustivamente la materia de la justicia sobre pertenencias:

1. Una persona que adquiere una pertenencia, de conformidad con el principio de justicia en la adquisición, tiene derecho a esa pertenencia.

2. Una persona que adquiere una pertenencia de conformidad con el principio de justicia en la transferencia, de algún otro con derecho a la pertenencia, tiene derecho a la pertenencia

3. Nadie tiene derecho a una pertenencia excepto por aplicaciones (repetidas) de 1 y 2).

El principio completo de justicia distributiva diría simplemente que una distribución es justa si cada uno tiene derecho a las pertenencias que posee según la distribución”26.

Según Nozick, su teoría retributiva de la justicia se desarrolla en tres reglas o principios ${ }^{27}$ :

\footnotetext{
${ }^{24}$ KYMLICKA, Filosofía política contemporánea, cit., p. 122 y 123.

${ }^{25}$ Puede verse una interesante y esclarecedora versión de la teoría intitulacionista de Nozick EN R. J. VAN DER VEEN y PH. VAN PARIJS, "Teorías intitulacionistas de la justica. De Nozick a Roemer y más allá”, Ideas y valores. Revista Colombiana de Filosofía, vol. 62, no 152, 2013, pp. 249-265. También F. SCHEMBER, "La teoría del título válido de Robert Nozick: un balance", Estudios públicos, 140 (primavera 2015), pp. 55-82.

26 Para todas las afirmaciones de NOZICK $A S U$, p. 154-155.

$27 A S U$, p. 154 y ss.
} 
1.- Un principio de justicia en las adquisiciones, por el cual se explica cómo se adquieren los bienes y recursos originariamente. Los intercambios del mercado requieren que las "pertenencias" estén debidamente justificadas, esto es, que los bienes o recursos objeto del mismo se posean con justo título y la justicia del mismo depende de que inicialmente, en un primer momento, se poseyera en fair play. Ni el robo, ni el fraude, ni el engaño o cualquier otro tipo de extorsión sirven como títulos justos. La justicia del título depende de que originariamente se adquiera la posesión justamente. Como he transcrito, para Nozick, "una distribución es justa si surge de otra distribución justa a través de medios legítimos".

Este primer principio que desarrolla la máxima inicial resulta bastante controvertido, pues ¿cómo se produjo el momento de la posesión de los bienes naturales, eran res nullius y se las apropió el primero que dijo "esto es mío" y los demás lo aceptaron? Nozick retrotrae la validez de su teoría a ese primer momento, ese estado de naturaleza al estilo de Locke, en el que, si bien no se formalizó en un contrato social, sin embargo, se produjo la apropiación de los bienes naturales y los recursos con importantes consecuencias para la posteridad. La descripción de Nozick no deja de representar una visión sobre los primeros momentos de "la conquista del oeste" en la que las grandes extensiones de tierra aparecían libres y dispuestas para que vaqueros como J. Wayne se apropiaran "injustamente" pues nadie tuvo en cuenta la existencia y los títulos de los indígenas ${ }^{28}$. En fin, conviene no perder de vista que la teoría de Nozick es una teoría a posteriori hecha para el sector de la población norteamericana que triunfó e impuso sus reglas.

2.-Un principio de transferencia, por el cual las pertenencias que sean justamente adquiridas pueden ser libremente transferidas. Este principio señala cuáles son los procedimientos justos de transmisión de los bienes y, por tanto, de la adquisición justa. Mi justo título sobre un bien depende, según Nozick, de que las diferentes transmisiones realizadas hasta llegar a la posesión actual hayan sido realizadas a través de procedimientos justos. Es, pues, un criterio de justicia histórica y temporal. Supone que la propiedad anterior hasta llegar al momento presente tiene un título justo, y así sucesivamente. Y mi derecho sobre ese bien será tan amplio, o sujeto a limitaciones, como la amplitud del derecho que me los transmitió, y así sucesivamente en el tiempo.

3.- Un principio de rectificación de la injusticia, por el cual explica cómo corregir los efectos injustos de una adquisición o transacción incorrecta. En la historia de un título puede haber violaciones a los dos principios anteriores. Por este principio se trata de establecer una vía que permite rectificar los errores en el título habidos ya sea en la adquisición inicial o en las sucesivas transmisiones. Plantea qué debe hacerse en la actualidad ante estas situaciones para subsanar un título injusto, las obligaciones de quienes cometieron la injusticia, cómo compensar el daño producido, etc.

El que un individuo tenga la propiedad de un bien con justo título depende no sólo de que las transferencias últimas realizadas sean justas, sino de que también la adquisición inicial también lo sea. "Los lineamientos generales de la teoría de la justicia de pertenencias son que las pertenencias de una persona son justas si tiene derecho a ellas por los principios de justicia en la adquisición y en la transferencia, o por el principio de rectificación de la injusticia”29.

\footnotetext{
${ }^{28}$ Esta imagen de sujetos a lo John Wayne apropiándose de terrenos aparentemente res nullius, no es de mi cosecha. Aparece en GARGARELLA, La teoría de la justicia después de Rawls, cit., pp. 52-53. Parece bastante apropiada para lo que NOZICK tiene en mente y para un sector de la población estadounidense, incluso hoy en día.

${ }^{29} A S U$, p. 156.
} 
Esta descripción de los principios de justica, de su teoría sobre el derecho de propiedad plantea serias dudas. Primero de todo, dudas sobre la importancia de la adquisición inicial; además, sobre la viabilidad del principio de rectificación. Si la validez de mi título sobre un bien depende de la validez de los títulos y derechos de propiedad anteriores, es fundamental que nos remontemos en la cadena de transmisiones hasta el momento inicial de apropiación; deberemos juzgar si ésta fue poseída justamente. Si esa primera apropiación fue injusta, el propietario no tiene un derecho justo sobre este bien y, por tanto, no puede transmitirlo legítimamente. La legitimidad del título y la inicial adquisición justa adquiere así en Nozick una importancia superlativa, pues es, a la postre, lo que legitima los intercambios del mercado y los beneficios y riquezas que las personas pueden obtener.

He aquí un serio problema para la teoría de Nozick, pues históricamente está probado que los sucesos originales no fueron tan armoniosos, que la fuerza y la violencia fue, al menos, uno de los modos de apropiación inicial, y la fuerza es un medio ilegítimo de adquisición. Si la primera apropiación es ilegítima, entonces todas las transmisiones hasta el momento presente se basan en un título injusto. Por otra parte, la teoría de Nozick parece pensada sólo para pertenencias duraderas, como bienes inmuebles, y no parece que encajen los bienes perecederos que, sin embargo, son producto de numerosos intercambios en el mercado.

Sigue siendo, no obstante, un escollo difícil de superar la cuestión de qué es lo que hace incuestionable el título y los derechos de la adquisición inicial de un bien. Su respuesta es una referencia a la teoría de Locke -la "estipulación de Locke"- sobre la apropiación y los cercados de tierras que se produjeron en Inglaterra en el siglo XVI. En última instancia, pretende convencernos de que, en tiempos pretéritos -en el momento de un estado de naturaleza- la tierra y los recursos del planeta no eran de nadie y que, en consecuencia, se podía adquirir un derecho legítimo e incuestionable si ello no empeoraba la situación de nadie. A partir de ahí se hace necesario un mercado libre que encauce la transmisión de bienes, capital y trabajo.

El estado de naturaleza cobra así una especial importancia para justificar su teoría sobre la propiedad, lo que es decir sobre los derechos y la concepción de la persona como dueño de sí misma, y el mercado. Y su teoría sobre el Estado mínimo. Pero, Nozick reelabora la descripción de Locke de esa primera apropiación para dar forma a la denominada "estipulación de Nozick" que cobra así una especial importancia en el conjunto de su teoría retributiva de la justicia.

\section{Reconstrucción histórico-antropológica de la historia institucional y política}

El retorno a los orígenes es una estrategia central en la fundamentación de la teoría de Nozick. De hecho, éste presta una especial atención a reconstruir la historia de la humanidad desde el estado de naturaleza hasta el Estado mínimo ${ }^{30}$. Por ello, si esa reconstrucción es correcta y, por tanto, convincente, Nozick logra un importante argumento en favor de su teoría.

El proceso histórico se compone de cuatro fases: el estado de naturaleza, la emergencia de las asociaciones de protección, el Estado ultraminimo y, finalmente, el Estado minimo. El proceso es como sigue: "De la anarquía, a través de las agrupaciones espontáneas, las asociaciones de ayuda mutua, la división del trabajo, las relaciones del mercado, las economías de escala y el autointerés racional, surge algo

30 "La justicia de pertenencias es histórica; depende de lo que, en realidad, ha ocurrido" ASU, p. 155. 
que se parece a un Estado mínimo o un grupo de Estados mínimos geográficamente delimitados"31. $\mathrm{Y}$ el mecanismo que permite el paso de uno a otro es un mecanismo de mano invisible.

El estado de naturaleza de Nozick pretende ser una revisión mejorada del estado de naturaleza de Locke. Según Locke, los individuos en el estado de naturaleza se encuentran en un estado de perfecta libertad y disponen de sus bienes como desean dentro de los límites del derecho natural. Esto exigen que se respete la vida, salud, libertad y posesión de los demás. La existencia de transgresores en el estado natural y la necesidad de defenderse y castigar estos actos. -los "inconvenientes del estado de naturaleza" de Locke ${ }^{32}$ - exigen un remedio: este es el gobierno civil.

Para Nozick, el Estado no tiene por qué ser el remedio más apropiado: "Tenemos que considerar también qué arreglos podrían hacerse dentro de un estado de naturaleza para enfrentar estos inconvenientes... Únicamente después de que todos los recursos del estado de naturaleza sean puestos en juego, a saber: todos aquellos arreglos y acuerdos voluntarios que las personas pudieran realizar en el ejercicio de sus derechos, y sólo después de que los efectos de éstos sean considerados, estaremos en posición de apreciar cuán serios son los inconvenientes que aún quedan por remediar por el Estado y estimar si el remedio es peor que la enfermedad" 33 .

Según Nozick, efectivamente, la aplicación de los derechos "conduce a contiendas, a series interminables de actos de represalia y de exigencias de compensación. Y no hay un medio seguro de resolver tal disputa, de terminarla, y hacer que ambas partes reconozcan que ha terminado" 34 . Ahora bien, el remedio no es el gobierno civil. Más bien, según la lógica interna del propio estado de naturaleza, el remedio será, primero, la defensa individual de los derechos y, pronto, la unión de individuos con el mismo objetivo. El primer paso relevante es, por tanto, la formación de asociaciones de protección. "Grupos de individuos pueden formar asociaciones de protección mutua: todos responderán a la llamada de cualquier miembro en defensa o exigencia de sus derechos. La unión hace la fuerza" 35 . Las asociaciones de protección mutua responderían a las agresiones externas a los derechos de los miembros y además arbitraría los procedimientos de resolución de los conflictos que surgieran internamente.

El éxito o fracaso en la defensa ante las agresiones externas y en la resolución de los conflictos internos acarrearía que otros individuos deseen sumarse a la asociación de protección mutua. Con el tiempo, estas asociaciones perfeccionarían sus métodos e incluso adquirirín mayor complejidad. Introducción entonces el principio de división de trabajo por el que alguno de los miembros se dedicaría a la función de protección y no haría falta el recurso a la llamada general de autodefensa. Algunos miembros se convertirían en empresarios creando empresas cuya función sería la de protección. Esta se convertiría en un servicio por el que hay que pagar. "La inconveniencia de que todos sean llamados cualquiera que sea su actividad en el momento ... puede ser manejada de la manejada usual: por la división del trabajo e intercambio. Algunos serán contratados para realizar funciones de protección y algunos empresarios entrarán en el negocio de vender servicios de protección. Se ofrecerán diferentes tipos de protección, por diferentes precios, a aquellos que pudieran desear una protección más amplia o más elaborada" 36.

\footnotetext{
31 ASU, p. 29.

32 ASU, p. 23.

33 ASU, p. 23.

34 ASU, p. 24.

35 ASU, P. 25.

${ }^{36} A S U$, p. 26.
} 
De esta forma y con el tiempo, surgen agencias protectoras a modo de empresas cada vez más complejas que prestan sus servicios a los individuos particulares a cambio de un precio. Lo curioso del caso es que los individuos no renuncian, a pesar de contratar los servicios de una agencia protectora, a la venganza privada. Nozick plantea diferentes situaciones que se pueden producir cuando aparecen conflictos entre particulares y estos reaccionan expeditivamente. Finalmente explica cómo poco a poco en una misma área geográfica surgirá una asociación de protección dominante.

En este caso, en su opinión, emerge ya un embrión de Estado con funciones de protección, de arbitraje y de resolución de conflictos. Cuando en una misma área coexisten varias asociaciones de protección pueden producirse tres situaciones: 1.- Que una sea más efectiva en la protección que las demás; entonces, los clientes irán pasándose a la más eficiente. 2.- Que dos asociaciones de protección dominen en áreas geográficas diferentes y cada una sea más eficiente en la prestación de sus servicios a medida que nos acerquemos al centro de su espacio de actuación. 3.- Que dos agencias eficientes y poderosas entren en conflicto; según Nozick, no hay ninguna razón para pensar que no se pondrán de acuerdo para que una tercera instancia arbitre sus diferencias. Se implanta así un sistema judicial federal unificado que resuelve las querellas entre asociaciones de protección ${ }^{37}$.

En este momento, nos encontramos en una situación cuasi-estatal. El Estado aparecerá cuando una de las asociaciones dominantes monopolice en un territorio el uso de la fuerza y las funciones de protección y eso sucederá cuando desaparezcan o absorba las demás asociaciones de protección y cuando los posibles independientes que queden en el territorio engrosen la lista de clientes. Para Nozick, no existen grandes dificultades para que esto suceda. Entonces, se constituye lo que llama el Estado ultramínimo, "que mantiene un monopolio sobre todo el uso de la fuerza, con excepción del que es necesario para la inmediata defensa propia y, por tanto, excluye la represalia privada (o la proporcionada por una agencia) por daño y para exigir compensación. Sin embargo, únicamente ofrece protección y servicios de ejecución a aquellos que compran sus pólizas de protección y aplicación. Las personas que no contratan protección con el monopolio no obtienen protección" 38 .

El Estado mínimo surge cuando el Estado ultramínimo se extiende, incluso coactivamente, a todas las personas que habitan en un territorio incluyendo aquéllas que no habían contratado la póliza de protección. El Estado mínimo es el Estado gendarme por antonomasia que monopoliza el uso de la fuerza y las funciones de protección en un territorio incluso contra la voluntad de las personas que no contrataron las pólizas iniciales. El Estado mínimo así constriñe a éstos a admitir su autoridad. Además, es un Estado redistributivo "hasta el grado de que constriñe a ciertos individuos a pagar por la protección de otros" 39 .

Según Nozick, todos estos pasos y transiciones obedecen a explicaciones de mano invisible, al estilo de las proporcionadas por A. Smith. Rechaza, por tanto, las explicaciones proporcionadas por Locke, Rousseau, Kant o Rawls. Los tránsitos entre estadios no se producen ni por contrato, ni pacto, ni consentimiento. No hay planificación, ni diseño racional. Se produce por la propia evolución de las cosas como si fuesen movidas por una "mano invisible". "Una explicación de mano invisible explica lo que parece ser el producto del designio intencional de alguien, como no causado por la intención de alguien" ${ }^{40}$. Las sucesivas transiciones desde la anarquía inicial hasta el Estado mínimo,

\footnotetext{
37 ASU, p, 28-29.

38 ASU, p. 39.

39 ASU, p. 39.

40 ASU, p. 32.
} 
por tanto, no se producen por un acto de consciencia humana, nadie lo busca conscientemente, sino que se produce de forma espontánea por la concatenación de ciertos factores.

La explicación de la mano invisible justifica el surgimiento del Estado, pero, sobre todo, que se realiza sin violencia a los derechos individuales. Los derechos individuales y, por tanto, la consideración de ser dueños de sí mismo queda así intacta incluso dentro del Estado mínimo. La idea es que esos individuos dueños de sí mismo y portadores de derechos absolutos, en determinadas circunstancias, puede considerar conveniente e interesante la formación de asociaciones de protección y así sucesivamente hasta llegar al Estado mínimo. Con ello, también pretende mostrar que su teoría está plenamente justificada tanto en relación con los Estados distributivos, tipo de Rawls, que, en su opinión, violan derechos, como frente a la postura anarquista tradicional, defensora del no Estado. Frente a los anarquistas, considera que algún tipo de Estado es necesario, que es mejor que la anarquía inicial, donde todos están expuestos a la violencia de los demás, y a las asociaciones de protección, que exigen demasiados esfuerzos y resultan costosas.

Igualmente, una explicación del tipo de la mano invisible reafirma el papel del mercado en el surgimiento del Estado mínimo. Un mercado que funciona sin control estatal, pero que beneficia a las asociaciones de protección más eficientes que se convierten así también en más poderosas. Dicho de otra forma, la evolución y el tránsito de una fase a otra se produce como efecto del mecanismo de la oferta y la demanda de protección donde la eficacia en la dotación de seguridad en la vida y en los bienes y el precio son dos factores determinantes en el éxito o fracaso de una asociación y en el hecho de que una de ellas acabe monopolizando el uso legítimo de la fuerza y los poderes del mercado.

\section{Justicia retributiva vs. Justicia distributiva}

Como señalé al principio, la obra de Nozick, Anarquía, Estado y Utopía, su primera obra y dedicada a la filosofía política, tiene su origen en el objetivo de establecer una alternativa, dentro del liberalismo, al éxito de $A$ Theory of Justice de J. Rawls. Si ésta, en los años 70s, refleja el ideario del igualitarista, la obra de Nozick expresa el modelo del anarquismo libertario de la justicia, de la sociedad y del Estado. En consecuencia, no podía faltar en Anarquía, Estado y Utopía la referencia a la obra de Rawls y a su modelo de justicia distributiva.

Precisamente, la Segunda Parte del libro de Nozick se abre con un capítulo VII dedicado a "La justicia distributiva". En él, incluye una segunda sección sobre "La teoría de Rawls"41. Nozick juega con una ilusión: el libro tiene por objeto mostrar la validez del anarquismo libertario frente al liberalismo social y, sin embargo, aparentemente sólo se refiere marginalmente a su mayor defensor y rival, en una segunda sección de este capítulo.

Esta parte comienza con la conocida frase: "El Estado mínimo es el Estado más extenso que se pueda justificar. Cualquier Estado más extenso viola los derechos de las personas" ${ }^{2}$. A continuación, en la primera sección Nozick desarrolla su teoría retributiva que, como hemos visto, gira en torno a su "teoría de título justo" y sus principios sobre la adquisición original de pertenencias, el

\footnotetext{
${ }^{41}$ Esta sección se inicia con un párrafo en el que Nozick hace una valoración altamente positiva del libro de Rawls: "A Theory of Justice es un trabajo vigoroso, profundo, sutil, amplio, sistemático dentro de la filosofía política y la filosofía moral como se había visto otro igual cuando menos desde los escritos de John Stuart Mill. Es una fuente de ideas esclarecedoras, integradas en un todo perfecto". Para continuar afirmando la manida frase de que "ahora los filósofos políticos tienen que trabajar según la teoría de Rawls, o bien, explicar por qué no lo hacen” (ASU, p. 183).

42 ASU, p. 153.
} 
de la transmisión de pertenencias y el de rectificación de las pertenencias en la injusticia. Estos principios constituyen su teoría retributiva de la justicia. Realmente, en su opinión, una teoría retributiva materializa una visión distributiva de la justicia, pues, como ya transcribí, en su opinión, "el principio completo de justicia distributiva diría simplemente que una distribución es justa si cada uno tiene derecho a las pertenencias que posee según la distribución. Una distribución es justa si surge d otra distribución justa a través de medios legítimos" ${ }^{43}$. Nada más lejos de la teoría de la justicia distributiva defendida por Rawls.

La justicia como equidad de Rawls se estructura en torno a dos principios fundamentales: el principio de igual libertad, que garantiza un esquema de derechos e igual libertad para todos, y un segundo principio que se subdivide en el principio de la diferencia y el principio de igualdad de oportunidades, con los que pretende materializar un ideal de igualdad real que rectifique las desigualdades económicas y sociales. A estos principios, se añade el "orden lexicográfico" por el que Rawls establece una jerarquía entre el primer y segundo principio y, dentro de este, entre el segundo y primer apartado del siguiente principio. Establece así un esquema rígido de materialización de su ideal de justicia distributiva ${ }^{44}$.

La crítica de Nozick a la teoría de la justicia de Rawls parte de la puesta en cuestión del modelo de cooperación social y de la necesidad intrínseca de la justicia distributiva. "¿Por qué la cooperación social crea el problema de justicia distributiva? ¿No habría ningún problema de justicia ni necesidad de una teoría de justicia, si no hubiera en absoluto ninguna cooperación social, si cada persona obtuviera su parte únicamente por sus esfuerzos propios?... ¿en virtud de qué hechos de cooperación social surgen estas cuestiones de justicia? ¿Qué hay en la cooperación social que origina cuestiones de justicia?" 45 . Vamos, que la cooperación social es una filfa, un invento para restringir derechos y justificar un Estado intervencionista.

Efectivamente, "la cooperación social enturbia las aguas haciendo que no sea claro o que sea indeterminado quién tiene el derecho a qué". Más bien, el modelo, según Nozick, es el de los Robinsones Crusoes cada uno trabajando en una isla y desarrollando su vida sin la necesidad de relacionarse con otros Robinsones. El ejemplo es típico de la fantasía de Nozick. Considera que diez Robinsones pueden vivir en islas separadas durante años y desarrollar su vida de acuerdo a sus capacidades naturales..., y que sobrevivirán. Esto demuestra que la tesis de las comunidades aisladas, de la responsabilidad individual, de la primera adquisición y de la transferencia de pertenencias, y del mercado es suficiente; es la justificación de la justicia retributiva y de que todo juega en contra de la teoría de Rawls ${ }^{46}$. Pura fantasía.

En realidad, Nozick está defendiendo que, si no hay relaciones humanas, como en el cuento de Robinson Crusoe ${ }^{47}$, no hay cooperación social -lo que quiere decir que no hay relaciones sociales, entonces no es necesaria la justicia distributiva. Esto es, sin duda, un "potente" ataque a la línea de flotación de la justicia como equidad.

A partir de estas consideraciones, Nozick critica la posición original, la elección de los principios de la justicia como equidad y, sobre todo, el principio de la diferencia. La base de la crítica

\footnotetext{
43 ASU, p. 154.

${ }^{44}$ Para un análisis más detallado de la obra de J. RAWLS puede verse J. MARTÍNEZ DE PISÓN, "Una lección sobre la justicia como equidad de John Rawls", Revista Electrónica del Departamento de Derecho de la Universidad de La Rioja (REDUR), 18, 2020, pp. 3-76.

45 ASU, p. 184-185.

46 ASU, p. 185.

${ }^{47}$ Lo que, por otra parte, no es cierto si atendemos a la relación vertical con Viernes.
} 
a este último se basa en la cuestión de que no es creíble que los menos aventajados puedan esperar la cooperación voluntaria de los más aventajados: "¿es justo un acuerdo sobre cuya base los peor dotados podrían esperar la cooperación voluntaria de otros?”. O, en otro sentido, “¿cuánto gana cada individuo de la cooperación social general, comparado, no con no cooperación más limitada?”. De nuevo, la conclusión parece clara: lo mejor es limitar la cooperación desde arriba y que toda relación social sea voluntaria al modo de los Robinsones, como si este ideal fuese posible en un mundo globalizado. Estas reflexiones no dejan de ser consecuencia de sus tesis iniciales sobre la gestación históricoantropológica del Estado mínimo: del individuo aislado a las asociaciones de protección, el Estado ultramínimo y, finalmente, el Estado mínimo. Y así Nozick acusa a Rawls de incongruencia al defender la mejora de los menos aventajados, pues concluye que "lo que se sigue en realidad... es una profunda desconfianza de imponer, en nombre de la imparcialidad, restricciones a la cooperación social voluntaria ( $\mathrm{y}$ al conjunto de pertenencias que surgen de ella), de manera que los que ya se benefician más de esta cooperación general se beneficien aún más" ${ }^{48}$. De hecho, Nozick considera que sus principios de justicia histórico-retributiva no saldrían muy bien parados en el contexto de debate, justificación y decisión que es la posición original ${ }^{49}$.

En suma, "el principio de la diferencia es, ante todo, injusto" 50.

\section{VI. ¿E1 estado mínimo como la utopía del anarquismo libertario?}

Nozick dedica la tercera parte de Anarquía, Estado y Utopía a intentar convencer al lector de que Estado mínimo, a pesar de ser un "Estado real", cumple con las reglas básicas para constituir una utopía por la que luchar ${ }^{51}$. Lo hace, como en otras partes de su obra, no tanto dando argumentos, sino dando las oportunas explicaciones en las que intenta mostrar que cumple las condiciones para ser considerado el mejor de los Estados posibles. Esta parte del libro, que constituye la "utopía", es, curiosamente, una parte más bien breve del texto en la que pretende establecer el "marco" en el que se puede realizar la utopía libertaria. Su objetivo es, pues, justificar que el Estado mínimo cumple con esas condiciones, con ese modelo de utopía.

Nozick parte de una aclaración importante sobre lo que es "utopía" que poco tiene que ver con los modelos antiguos al estilo de Th. Moro y lo mismo puede afirmarse respecto a las propuestas más actuales. En realidad, cuando Nozick hace referencia a la utopía se refiere "al mejor de los mundos posibles": pues "la totalidad de condiciones que nos gustaría imponer a las sociedades que deben (preeminentemente) ser calificadas de utopía, tomadas en su conjunto, son inconsistentes" ${ }^{52}$.

\footnotetext{
48 ASU, p. 193.

${ }^{49}$ Como afirma Nozick, "todo el procedimiento de las personas que escogen principios, en la posición original de Rawls, presupone que ninguna concepción histórico-retributiva de justicia es correcta” (ASU, p. 200). Esta idea encaja perfectamente con su idea de que la emergencia del Estado mínimo no es una cuestión de principios, sino de decisiones personales realizadas por individuos pragmáticos.

50 ASU, p. 202.

${ }^{51}$ Esta tercera parte de $A S U$ comienza del siguiente tenor: "Ningún Estado más extenso que el Estado mínimo se puede justificar. Pero, ¿no carece de lustre la idea, o el ideal, del Estado mínimo? ¿Puede llegar al corazón o inspirar a las personas para que luchen o se sacrifiquen? ¿Habría alguien que excavara barricadas bajo su bandera?..." (ASU, p. 287).

$52 A S U$, p. 287.
} 
"La utopía debe ser, en algún sentido restringido, la mejor para todos nosotros; el mejor mundo imaginable, para cada uno de nosotros" ${ }^{\prime 5}$.

En base a la idea de que, en el mundo real, los individuos entran y salen de comunidades, de que son admitidos o excluidos de las mismas y de que deben o no soportar restricciones, Nozick diseña tanto un "modelo" como un "marco" para la utopía. Su modelo de utopía -de "mundos posibles"- está basado en la idea de que, en el mundo, los seres racionales viven en comunidades, "asociaciones estables", de las que pueden salir y entrar, en las que pueden tener que soportar restricciones o no, en las que pueden ser expulsados o no ${ }^{54}$. Por el contrario, el "marco" hace referencia a las circunstancias concretas del mundo real al cual tiene que adaptarse este vago modelo de utopía. En concreto, cuatro 55 : 1.- En el mundo real, "no podemos crear todas las personas cuya existencia deseamos". 2.- "En el mundo real las comunidades tropiezan una con la otra, creando problemas de relaciones exteriores y autodefensa y necesitando formas de decisión judicial y de resolución de disputas entre comunidades". 3.- Igualmente, "hay costos de información para descubrir qué otras comunidades hay, y cómo son, así como costos de traslado y de viaje para ir de una comunidad a otra". 4.- Aún más, "algunas comunidades pueden tratar de mantener ignorantes a algunos de sus miembros sobre la naturaleza de otras comunidades a las que ellos podrían unirse, para tratar de impedirles abandonar libremente su propia comunidad y unirse a otra". Esto genera el problema del establecimiento de procedimientos que garanticen le libertad de movimientos, según Nozick.

Cómo, a partir de ese modelo y de ese marco se alcanza el mejor de los "mundos posibles" en la realidad -es decir, un conjunto de asociaciones bajo el Estado mínimo- "constituye un campo para la aplicación de las teorías más desarrolladas que tratan de la decisión de agentes racionales (a saber: teoría de la decisión, teoría de los juegos y análisis económico), instrumentos que seguramente tienen que ser de importancia para la filosofía política y la ética" 56 .

A partir de ahí, las explicaciones de Nozick adquieren altas dosis de complejidad. Con el objeto de realizar el modelo, el autor de Anarquía, Estado y Utopía considera que hay tres rutas para que, en el seno del marco descrito, pueda alcanzarse la utopía del Estado mínimo. Lo importante de sus explicaciones es que considera que el proceso estaría determinado por dos métodos que denomina "mecanismos de diseño" y "mecanismos de filtro" 57.

Por el mecanismo de diseño se construye algo. En el caso de una sociedad, "el resultado del proceso del diseño es una descripción de una sociedad, lograda por personas (o una persona) que se sientan y piensan sobre cuál es la mejor sociedad. Después de decidir, se ocupan de basarlo sobre este modelo". Pero, a estas alturas de la evolución de la humanidad y de la sociedad, que un mecanismo como éste tuviese éxito es muy improbable. Máxime si se tiene en cuenta la complejidad y variedad del ser humano, de las relaciones interpersonales, así como de las instituciones sociales y de las organizaciones. Resulta ingenuo imaginar un momento pasado, presente o futuro que tal mecanismo de diseño pudiera haberse materializado.

\footnotetext{
53 ASU, p. 288.

54 ASU, p. 289.

55 ASU, p. 296.

56 ASU, p. 295.

${ }^{57} A S U$, p. 300 y ss.
} 
Por eso, entran en funcionamiento los "mecanismos de filtración": "presuponen un proceso que elimina (filtra) muchas alternativas de un conjunto grande de alternativas" 58 . De esta manera, a través de estos mecanismos de filtración, puede llegar a concretarse el diseño de la sociedad realizada por los diseñadores. Para ello, según Nozick, resulta importante "la naturaleza particular del proceso de filtración" y "el conjunto de alternativas sobre las que opera".

Nozick resume el proceso de diseño y materialización de la sociedad de un modo que recuerda a Rawls: "Un proceso de filtración para especificar una sociedad que se nos podría venir a la mente es uno en que las personas que planean la sociedad ideal consideran muchas clases diferentes de sociedades y critican algunas, eliminan algunas, modifican las descripciones de otras, hasta que llegan a la que consideran la mejor". No obstante, el proceso poco tiene que ver con la posición original y el juego de regateo de Rawls. En efecto, según Nozick, el proceso de filtración -o de selección- es muy sencillo. Se trata de que las personas entren y salgan de las asociaciones o comunidades, de que intenten mejorarlas o no, de la evolución y desarrollo de cada una de ellas: unas desaparecerán, otras se dividirán o fortalecerán, etc. De manera que cada comunidad tiene un objetivo claro: "ganar y mantener la adhesión voluntaria de sus miembros" 59.

En fin, la interrelación entre el "marco" de la utopía y los "mecanismos de filtración" garantizan la correcta realización de la utopía libertaria. Nozick, en epígrafe titulado "El marco como el fundamento común utópico", afirma con sensación de triunfo: "El uso de un mecanismo de filtro que depende de las decisiones individuales de las personas, de vivir e o dejar comunidades particulares es especialmente apropiado. Puesto que el propósito último de la construcción utópica es lograr comunidades en que las personas querrán vivir y en las que escogerán vivir voluntariamente $\mathrm{o}$, al menos, éste tiene que ser un efecto colateral de la buena construcción utópica" ${ }^{60}$. Y la base del éxito está en que las personas, a veces, tenemos dificultades en esbozar y pergeñar principios que permitan anticipar situaciones complejas; sin embargo, no ocurre lo mismo cuando se toman decisiones apegadas a la realidad de cada sujeto. Este último reúne las ventajas de "un mecanismo de filtración que dependa de las decisiones de las personas" frente a los procesos inspirados en principios.

Nozick confía en convencernos de que su esquema teórico, especialmente, la relevancia del "marco", es el correcto, sobre todo, si tenemos en mente el logro de una comunidad que no se basa en los principios, sino en las decisiones personales, que es lo que tiene en la mente. A pesar de lo difuso del (supuesto) marco, considera los argumentos a su favor que "son aún más fuertes cuando abandonamos el (falso) supuesto de que hay una sola clase de sociedad que es la mejor para todos y dejamos de interpretar mal el problema como uno en el que hay una sola clase de comunidad en la cual toda persona debe vivir". Y, en consecuencia, afirma que "el marco tiene dos ventajas sobre cualquiera otra clase de descripción de utopía: primera, será aceptable para casi cualquier utopista en algún momento en el futuro, cualquiera que sea su visión particular; y segunda, es compatible con la realización de casi todas las visiones utópicas particulares, aunque no garantiza la realización o el triunfo universal de ninguna visión utópica particular" ${ }^{1}$.

Una de las críticas que se ha hecho a Rawls, como vimos en su capítulo, es que todo el imaginario conceptual tiene el único objetivo de justificar y legitimar sus principios de la justicia como equidad. Buena parte de sus aportaciones son un constructo elaborado con ese fin. En particular, sus

\footnotetext{
58 ASU, p. 301.

59 ASU, p. 303.

${ }^{60} A S U$, p. 305.

${ }^{61} A S U$, p. 306-307.
} 
reflexiones sobre la posición original, sobre todo, el diseño que se hace de esta situación y del funcionamiento del juego de regateo que debe conducir a la decisión final sobre los principios de justicia.

Lo mismo puede afirmarse de Nozick en esta ocasión. La idea del modelo, su diseño del marco y la interrelación con los mecanismos de diseño y de filtración deben conducir a la realización de una o varias comunidades cuyo amparo protector es el Estado mínimo. Resulta difícil imaginar que todo el conjunto de versiones de la "utopía" puedan acabar confluyendo en esta versión del Estado, del mismo modo que no es creíble que el enmarañamiento de los conceptos antes descritos pueda lugar en la utopía del Estado mínimo tal y como Nozick lo describe. Inspirado en la propuesta de Hayek, termina afirmando que "la postura expuesta aquí rechaza totalmente la planeación en detalle, participando de una comunidad en la que cada uno va vivir; sin embargo, simpatiza con la experimentación utópica voluntaria y le ofrece el ambiente en el cual puede florecer; ¿cae esta posición dentro del marco utópico o antiutópico"62.

La respuesta ya ha sido anticipada desde la primera línea del texto Anarquía, Estado y Utopía: "El marco para la utopía que hemo descrito es equivalente al Estado mínimo. El argumento de este capítulo comienza (y se mantiene) independientemente del argumento de las partes Primera y Segunda y converge en su resultado: el Estado mínimo, desde otra dirección" Y sigue para concluir: "Sostuvimos en la Primera Parte que el Estado mínimo es moralmente legítimo; en la Segunda Parte sostuvimos que ningún Estado más extenso podría ser moralmente justificado, que cualquier Estado más extenso violaría (violará) los derechos de los individuos. Este Estado moralmente favorecido, el único Estado moralmente legítimo, el único moralmente tolerable es, como ahora vemos, el que mejor realiza las aspiraciones utópicas de incontables soñadores y visionarios": "¿No es el Estado mínimo, el marco para la utopía, una visión sugestiva?”33.

\section{Análisis crítico}

La teoría de Nozick contiene, sin duda, interesantes aportaciones, ideas intuitivas sugerentes y un fuerte atractivo para las sociedades desarrolladas e individualistas cuyo modelo es el de los viejos conquistadores del Oeste americano. Cuando uno lee el libro de Nozick, Anarquía Estado y Utopía, no deja de venirle a la cabeza ciertos acontecimientos que han sucedido recientemente en la "democracia más perfecta" del planeta. Su justificación de que los derechos individuales absolutos están por encima de las instituciones y de las responsabilidades públicas, de que la defensa personal es obligación del propio sujeto, en el fondo, avalan lo que son, en mi opinión, actitudes y hechos que sorprenden en la tierra de la libertad. Desde la importancia del lobby de la asociación del rifle hasta $\cdots$

La teoría de Nozick ha sido ampliamente revisada. Kymlicka señala alguna de las más importantes. Entre otros ${ }^{64}:$ 1.- Que de sus premisas sobre la persona como dueño de sí mismo no se deriva exclusivamente su modelo de un derecho inviolable y absoluto de la propiedad. Lo cual es muy cierto pues la misma historia -incluso, la jurídica- de ese derecho muestra cómo se ha ido fraguando la idea de las limitaciones de este derecho, el carácter social del mismo. 2.- Que, en realidad, una teoría sobre la propiedad como la esgrimida por Nozick es compatible con otros modelos sociales y

\footnotetext{
$62 A S U$, p. 318.

${ }^{63}$ ASU, p. 319.

${ }^{64}$ W. KYMLICKA, Filosofía política contemporánea., cit., pp. 109-143.
} 
políticos vigentes, incluido el de Rawls. Esto es, que de su teoría sobre el individuo y sus derechos no se deriva necesariamente una justificación del Estado mínimo como único Estado posible. 3. Que su concepto de ser dueño de sí mismo da lugar a una visión incorrecta -e inmoral- del principio de igualdad. Más bien, de la desigualdad entre las personas, del dominio y explotación de unos sobre otros.

Hay más críticas que no sólo afectan a su concepto de persona moral, a su teoría de los derechos y al Estado mínimo, sino también a una pieza que cobra una especial importancia como es "la explicación de la mano invisible". Gargarella, por ejemplo, insiste también en el análisis de su retórica sobre la propiedad y la escasa capacidad de convencimiento de su teoría sobre el título justo, sobre todo, sus explicaciones sobre la adquisición inicial.

A lo largo del texto ya he desbrozado alguna de estas críticas. Creo especialmente relevantes las que afectan al momento de la adquisición inicial, a lo que se denomina "la estipulación de Nozick" como versión mejorada de "la estipulación de Locke". No resulta creíble un momento inicial en el que el mundo y los bienes materiales no sean de nadie y, de repente, por no se sabe qué razón "todo" es objeto de apropiación por unos pocos. Máxime cuando la condición que pone Nozick es que dicha apropiación no suponga "un empeoramiento" del resto. Esto no es creíble: cualquier apropiación perjudica al que queda desposeído por ese acto. Precisamente, una teoría de la justifica, si tiene alguna justificación en el marco de una economía de mercado, es para compensar los desequilibrios que pudieran producirse por estas apropiaciones y sus transferencias.

No me convence tampoco el recurso a "la explicación de la mano invisible" como el mecanismo orientador y, en suma, conductor de importantes procesos sociales, especialmente, de las transformaciones políticas que se producen en el tránsito del estado de naturaleza hasta alcanzar el Estado mínimo. Dicho expediente, el recurso a la mano invisible- no resulta tampoco creíble ni siquiera como componente de una teoría de la decisión racional que intente explicar el resultado de la interacción de voluntades particulares. Primero porque presupone una visión de la racionalidad humana ligada a la "razón instrumental" que, a estas alturas, ha sido hábilmente deconstruida ${ }^{65}$. Segundo, porque, utilizando este mecanismo, lo mismo puede originarse el Estado mínimo que lo contrario, y Nozick no afronta esta posibilidad: que, por el expediente de la mano invisible, de la interacción de decisiones y omisiones individuales, la estructura política acabe degenerando en un Estado totalitario. Existen ejemplos en la historia del último siglo de que, a pesar de todo, esto es posible.

Por último, el Estado mínimo no puede ser un ejemplo o modelo de un Estado inspirado en una teoría de la justicia. Puede estar inspirado en alguna teoría, pero no parece que esté correctamente fundado en una doctrina moral, racional y correcta, en una concepción equitativa de la justicia. Más bien, por su naturaleza, el Estado mínimo parece un Estado injusto, que fomenta las desigualdades e impide que sus ciudadanos puedan llevar una vida digna. Y, desgraciadamente, esta realidad no constituye una utopía.

\section{Bibliografía}

DIETERLEN, P., "La filosofía política de Robert Nozick”, Revista Mexicana de Ciencias Sociales y Politicas, vol. 37, no 150, 1998, pp. 123-135.

${ }^{65}$ Quizá haya que recordar en este punto el impresionante libro de Th. ADORNO y M. HORKHEIMER, Dialéctica del iluminismo. 
GARGARELLA, R., Las teorías de la justicia después de Rawls. Un breve manual de filosofía politica, Barcelona, Paidós, 1999.

GONZÁLEZ RICOY, I. y QUERALT, J., eds., Razones públicas. Una introducción a la Filosofía Política, Barcelona, Ariel, 2021.

GRONDONA, M., Los pensadores de la libertad. De Jobn Locke a Robert Nozick, Editorial Sudamericana, Buenos Aires, 1986.

JIMÉNEZ REDONDO, M., Constructivismo, Rawls, Nozick, Valencia, Servicio de Imprenta Universitaria, 1983.

KYMLICKA, W., Filosofía política contemporánea. Una introducción, trad. de R. Gargarella, Barcelona, Ariel, 1995.

LOCKE, J., Ensayo sobre el gobierno civil, trad. de A. Lázaro, Madrid, Aguilar, 1981,

MARTÍNEZ DE PISÓN, J., "Una lección sobre la justicia como equidad de John Rawls”, Revista Electrónica del Departamento de Derecho de la Universidad de La Rioja (REDUR), 18, 2020, pp. 3-76.

NOZICK, R., Anarquía, Estado y Utopía, trad. de R. Tamayo, México, FCE, 1988.

NOZICK, R., Explicaciones filosóficas, trad. de J. Marqués, Editorial Innisfree, Madrid.

NOZICK, R., Meditaciones sobre la vida, trad. de C. Gardin, Barcelona, Gedisa, 1992.

NOZICK, R., La naturaleźa de la racionalidad, trad. de A. Domenech, Barcelona, Paidós, 1995.

RAWLS, J., Teoría de la Justicia, trad. de M. D. González, Madrid, Fondo de Cultura Económica, 1979.

RENTMEESTER, C., "The Need for Basic Rights: A Critique of Nozick's Entitlement Theory”, SOCRATES: An International, Multi-lingual, Multi-disciplionary, vol 2, no 3, 2014, pp. 18-26.

RODILLA, M. A., "Buchanan, Nozick, Rawls. Variaciones sobre el estado de naturaleza", Anuario de Filosofía del Derecho, no 2, 1985, pp. 229-284.

SCHWEMBER, F., “La teoría del título válido de Robert Nozick. Un balance”, Estudios Públicos, 140, primavera 2015, pp. 55-82.

SCHWEMBER, F. y LOEWE D., "Si el mundo fuera completamente justo: Nozick y los límites de la utopía liberal", Revista de Estudios Políticos, 182, 2018, pp. 43-69.

SCHWEMBER, F. y LOEWE D., "No empeorar la situación de otros: la estipulación lockeana y las apropiaciones originarias del título válido de Nozick", Eidos, n 35, 2021, pp. 374-403.

VALLESPIN, F., Nuevas Teorias del Contrato Social: J. Rawls, R. Nozick y J. Buchanan, Madrid, Alianza Universidad, 1985.

VAN DER VEEN, R. J. y VAN PARIJS, Ph., "Teorías institucionales de la justicia. De Nozick a Roemer y más allá”, Ideas y Valores. Revista Colombiana de Filosofía, vol. 62, no 152, 2013, pp. $249-265$. 